\title{
Calidad de carcasas y cortes de cuatro biotipos de novillos en Corrientes, Argentina
}

\author{
Capellari, A. ; Rébak, G.I. ${ }^{2}$; Revidatti, M.A. ${ }^{\text {; }}$ Ynsaurralde, A.E. ${ }^{2}$ \\ ${ }^{1}$ Cátedra Introducción a la Producción Animal, ${ }^{2}$ Cátedra Tecnología de los Alimentos, Facultad de \\ Ciencias Veterinarias, UNNE, Sargento Cabral 2139, Corrientes (3400), Argentina. \\ Tel/Fax: 03783-425753. E-mail: acapellari@vet.unne.edu.ar.
}

\begin{abstract}
Resumen
Capellari, A.; Rébak, G.I.; Revidatti, M.A.; Ynsaurralde, A.E.: Calidad de carcasas y cortes de cuatro biotipos de novillos en Corrientes, Argentina. Rev. vet. 21: 1, 3-7, 2010. Con la finalidad de determinar caracteres previos a la faena, de faena y cortes comerciales de novillos de dos años de edad se evaluaron los biotipos: $3 / 8$ y 5/8 Cebú x Hereford ( $3 / 8$ y 5/8 $\mathrm{CxH})$, Hereford (TH) y Cebú (TC). En un diseño completamente aleatorizado, durante tres años consecutivos se seleccionaron diez novillos de cada biotipo, mantenidos sobre pastizal natural y pasturas cultivadas con suplementación estratégica, sacrificando los animales en frigoríficos tipo A. Previo al embarque, fueron pesados y evaluada la condición corporal (escala norteamericana 1-9). Luego del sacrificio se procedió a la clasificación y tipificación por conformación y grado de engrasamiento (ex-Junta Nacional de Carnes), obteniéndose pesos y rendimientos de faena. Luego de $24 \mathrm{~h}$ de maduración las medias reses izquierdas fueron cuarteadas y desosadas. Se obtuvieron pesos y rendimientos de cortes comerciales clasificados en categorías. No se encontraron diferencias significativas en peso vivo, condición corporal y peso de la res caliente en los novillos cruza, pero sí en rendimiento de faena. Entre los biotipos TH y TC se hallaron diferencias significativas para peso vivo, peso de res caliente y rendimiento de faena y éstos con respecto a los novillos $3 / 8$ y $5 / 8 \mathrm{CxH}$, arrojaron diferencias significativas para condición corporal entre los biotipos puros y sus cruzas. La categoría novillito fue la más frecuente para todos los biotipos, existiendo diferencias en conformación, grado de engrasamiento y peso de cortes de primera, donde los novillos $3 / 8$ $\mathrm{CxH}$ mostraron similar calidad de res y cantidad de $\mathrm{kg}$ de cortes que los TC siendo diferente en dichos parámetros los biotipos 5/8 CxH y TH. De acuerdo a los resultados obtenidos los cuatro biotipos constituyen alternativas válidas para obtener carcasas bovinas y cortes comerciales destinados al mercado consumidor adaptables a la región nordeste argentino.
\end{abstract}

Palabras clave: bovino, genotipo, rendimiento de carcasa, cortes de carne.

Capellari, A.; Rébak, G.I.; Revidatti, M.A.; Ynsaurralde, A.E.: Carcass and meat cuts quality of four steer biotypes in Corrientes, Argentina. Rev. vet. 21: 1, 3-7, 2010. To determine characters prior to slaughtering, slaughtering and commercial cuts of 2 years-old steers, animal carcass from biotypes 3/8 and 5/8 Zebu x Hereford, Hereford, and Zebu were analyzed. Under a complete randomized trial and during three consecutive years, ten steers from each biotype were fed on natural as well as artificial pastures with strategic supplementation. Prior to slaughtering, animals were weight and body condition score was determined ( 1 to 9 scale). Animals were slaughtered in a type A slaughterhouse. Carcasses were divided into two halves. Classification and typification were performed according to muscular development (shape) and thickness of fat covering muscles (fatness) obtaining weights and carcass yields. After $24 \mathrm{~h}$ left carcasses were cut on the $13^{\text {th }}$ rib and then boned. Boneless cuts were classified in different orders, getting individual weights and cut yield from the cold carcass. Differences among body live weight, body condition, and carcass weight were not statistically significant for Zebu crossbreed type. Yield grades were higher and without significance between 5/8 Zebu x Hereford biotype and Zebu type. Young steer categories (carcass less and $118 \mathrm{~kg}$ ) were the most frequent for all the biotypes. No differences were found in carcass quality, being them homogeneous. Fat levels were 1 and 2. First class cuts were higher in the $5 / 8$ Zebu x Hereford rather than the Hereford biotypes, but there were no differences for cut yields. According to these results, any of the four biotypes may be considered as excellent 
alternatives to obtain good quality in carcasses destined to the regional market of the northeastern region of Argentina.

Key words: cattle. genotype, carcass yields, meat cuts.

\section{INTRODUCCIÓN}

El avance de la agricultura provocó un reordenamiento territorial del rodeo nacional; en los últimos 16 años hubo un claro crecimiento ganadero en las regiones marginales acompañado de una disminución del stock en la región pampeana. En 1994, esta región contenía el $62,4 \%$ del stock, actualmente se estima $53 \%$. La región nordeste argentino (NEA) fue la principal beneficiada, pasando de tener $21,5 \%$ a $27 \%(14.388 .391$ cabezas) del stock nacional ${ }^{19}$.

En el NEA la composición genética del ganado vacuno comercial es cruza, participando razas indicas (Brahman) y británicas (en Corrientes predomina la raza Hereford), logrando así animales adaptados a la zona. En las décadas de 1980 y 1990 se determinó que la mejor vaca debía tener $3 / 8$ sangre cebú y $5 / 8$ sangre europea, produciendo terneros con carne de terneza aceptable para el mercado interno y externo. El resultado dio auge a las razas Braford y Brangus, que hoy predominan en la ganadería de la región ${ }^{1}$.

La información disponible sobre la ganancia de peso de novillos en campo natural del NEA, señala que se obtienen $124 \mathrm{~kg} /$ año entre los 8 y 20 meses de edad (de abril a abril) a una carga de 1 novillo/ha. A igual carga, el aumento de peso entre los 20 y 32 meses de edad es similar alcanzando $126 \mathrm{~kg} / \mathrm{anno}^{23}$. Esto es atribuido a la limitada calidad del forraje producido; los pastizales están compuestos por gramíneas $\mathrm{C} 4$ o tropicales, las que se caracterizan por un crecimiento estival, rápida maduración y una consecuente disminución de su calidad $5,14,15$.

En la región se ha observado una marcada tendencia a la utilización de grandes superficies de tierras para actividades agrícolas. Este hecho ha llevado a los productores a emplear diferentes estrategias. En el NEA las condiciones ambientales para proveer carne de alta calidad muchas veces es desperdiciada por la falta de utilización de tecnología disponible ${ }^{26}$.

Actualmente la calidad es un objetivo prioritario. Para el consumidor tienen especial importancia la calidad sensorial y la inocuidad del producto. Los criterios de calidad de carcasa consideran principalmente el rendimiento de res o de faena, a partir del cual los productores e industriales determinan el precio y las posibilidades de compra-venta. La tipificación vigente en Argentina desde la década de 1940, es un método de control de calidad que agrupa a las reses según categorías determinadas a partir de características comunes que influyen en su cotización y en la especificación de su destino industrial o de mercado ${ }^{2}$.

El estudio de la composición corporal de los bovinos tiene importancia desde distintos puntos de vista.
El más generalizado involucra la evaluación de las condiciones carniceras de la res para determinar su valor comercial. En este sentido es deseable un alto contenido de músculo, asociado a bajo porcentaje de tejido adiposo, pero dentro de valores tales que no afecten el grado de terminación y los caracteres organolépticos de la carne y por ende su aceptabilidad comercial.

El objetivo del presente trabajo fue determinar la influencia del biotipo, en novillos de dos años de edad $3 / 8$ y $5 / 8$ Cebú x Hereford ( $3 / 8$ y $5 / 8 \mathrm{CxH}$ ), Hereford (TH) y Cebú (TC), sobre caracteres previos a la faena, de faena y de cortes comerciales.

\section{MATERIAL Y MÉTODOS}

La cría y engorde de los animales se llevó a cabo en la Estación Experimental Agropecuaria del Instituto Nacional de Tecnología Agropecuaria (INTA, Corrientes, Argentina), ubicada a $30 \mathrm{~km}$ al sur de la capital de la provincia, entre el Río Paraná y la Ruta Nacional 12. El clima es subtropical sin estación seca, con temperatura media anual de $21^{\circ} \mathrm{C}$ y un régimen pluvial estival con medias anuales de $1.200 \mathrm{~mm}$.

Para la experiencia se utilizaron potreros de pastizal natural durante la época invernal y pasturas cultivadas de pasto pangola (Digitaria decumbens) el resto del año, con una carga animal promedio de 1,5 novillos/ha. Durante tres años se seleccionaron al azar diez terneros de cada biotipo de un sistema de cría bovina con servicio de primavera y destete de otoño, los cuales recibieron una suplementación de $1,5 \mathrm{~kg} / \mathrm{animal} / \mathrm{día}$ de pellets de trigo y semilla de algodón en igual proporción en la fase de crecimiento. En los últimos 80 días de engorde la semilla de algodón fue reemplazada por grano de maíz, lo cual permitió una ganancia de 400 y $800 \mathrm{~g} / \mathrm{animal} / \mathrm{día}$ en otoño-invierno y primavera-verano respectivamente, logrando tasas de crecimiento que permitieron la terminación para faena de los novillos a los 22 a 24 meses de edad.

Al finalizar el engorde los lotes de 40 animales de los cuatro biotipos fueron faenados en frigoríficos tipo A. Previo al embarque, se pesaron individualmente y se evaluó condición corporal (1: extremadamente flaco, 9: extremadamente gordo) ${ }^{3,12}$. Luego del sacrificio se procedió al romaneo, clasificación y tipificación ${ }^{20}$, calculándose los rendimientos de carcasa.

Después de $24 \mathrm{~h}$ de maduración las medias reses del lado izquierdo fueron cuarteadas y desosadas obteniéndose los cortes comerciales, los cuales fueron pesados individualmente, clasificándolos en categorías: cortes de primera, segunda y tercera, según calidad comercial, y se calcularon sus rendimientos. Los cortes vacunos categorizados de primera, denominados de "alta cali- 
dad o de exportación" fueron: cuadril, nalga de afuera (cuadrada y peceto), bola de lomo, lomo, bife ancho, bife angosto, nalga de adentro y carnaza de paleta. Los cortes categorizados de segunda (carniceros) fueron: asado con vacío y matambre, tortuguita, chingolo y aguja y los categorizados de tercera (de manufactura): garrón, brazuelo, cogote, pecho y recortes comestibles 2,20 .

Para cada variable paramétrica (peso vivo, condición corporal, peso de la carcasa, rendimiento de faena, peso total en $\mathrm{kg}$ de los cortes por categorías y rendimiento en porcentaje) se aplicó el análisis de la varianza (ANOVA) con un diseño completamente aleatorizado, teniendo en cuenta el efecto biotipo como fuente de variación. Para la comparación de medias se utilizó el test de Tuckey con un alfa $=5 \%$. Previo al análisis se verificaron los supuestos del ANOVA. Las variables no-paramétricas (categoría, tipificación y engrasamiento con alfa $=10 \%$ ) fueron analizadas por el test de Kruskal-Wallis y la comparación de medias se realizó por la prueba $\mathrm{K}-\mathrm{W}$.

\section{RESULTADOS Y DISCUSIÓN}

Caracteres previos y de faena. El efecto del biotipo afectó las variables peso vivo (PV), condición corporal (CC), peso de la media res caliente (PR) y rendimiento de carcasa (RC), logrando mayor cantidad de carne (peso res caliente) los biotipos cruza $3 / 8$ y $5 / 8$ CxH respecto a los definidos TH y TC (Tabla 1$)$.

En las variables PV, CC y PR los biotipos con $3 / 8$ y 5/8 de sangre Cebú no se diferenciaron significativamente. En cambio en el rendimiento de carcasa, el lote 5/8 CxH logró 1,26 unidades porcentuales más, diferenciándose así significativamente. Estos resultados coinciden con los de otros autores en cruza $3 / 8$ y $5 / 8$ Cebú x Angus ${ }^{4}$.

Entre los biotipos TH y $\mathrm{TC}$ hubo diferencias significativas en PV, PR y RC, excepto en CC, logrando el lote Cebú $30 \mathrm{~kg}$ más de peso vivo, 24,22 kg más de carne y 2,48 unidades porcentuales de rendimiento más que TH en iguales condiciones de producción primaria. Estos datos son relevantes ya que en la región no existe información comparable al respecto. El lote cebú logró un rendimiento de res de 0,43 unidades porcentuales no significativas con $5 / 8 \mathrm{CxH}$ y 1,8 unidades porcentuales con 3/8 CxH, estadísticamente significativas. Esto indica que al aumentar la sangre cebú aumenta el rendimiento en carcasa.

El efecto biotipo se manifestó en otros trabajos al encontrarse diferencias en el peso de carcasa y no en el peso vivo ${ }^{17}$, o en ambas variables ${ }^{22}$. Otros autores informaron para razas continentales, índicas y británicas pesos de carcasa mayores a los expuestos en este trabajo ${ }^{6,7,25}$. Se debe tener en cuenta el efecto del sistema de alimentación sobre las variables estudiadas: trabajos realizados con novillos Hereford sobre pasturas y con suplementación registraron pesos superiores en ambos casos ${ }^{18}$, al igual que en novillos británicos alimentados con dieta base de alfalfa y maíz, con adición de lípidos de distintas fuentes ${ }^{13}$.

El rendimiento de carcasa no difirió significativamente entre novillos $3 / 8 \mathrm{CxH}$ y TH $(0,68$ unidades porcentuales), al igual que entre los biotipos $5 / 8 \mathrm{CxH}$ y TC (0,43 unidades porcentuales); por el contrario, se registraron diferencias significativas entre novillos cruza Cebú de 1,37 unidades porcentuales y entre biotipos TH y TC de 2,48 unidades porcentuales, resultados ya informados por otros autores ${ }^{4,17}$, no siendo así en todos los casos ${ }^{16,27}$. Evaluando otros efectos, en otras investigaciones se han informado rendimientos similares ${ }^{8,9,13}$, y superiores $6,7,11$, en razas de distintos orígenes.

En la Tabla 2 se observa que la categoría mayoritaria, superior al $77 \%$, fue novillito (NT), que correspon- 
de a un peso de carcasa entre 75 y $117 \mathrm{~kg}$. Carcasas mayores a $117 \mathrm{~kg}$, que corresponden a la categoría novillos (NO) se obtuvieron en biotipos Cebú y cruzas, no así en TH, que por su menor peso de faena categorizaron ternero (T), que corresponde a carcasas menores de 75 kg. Entre 3/8 CxH y TC, la categoría no fue significativamente diferente, ocurriendo lo contrario entre 5/8 CxH y TH. La variable tipificación no difirió significativamente.

El grado de terminación fluctuó de manera similar a la variable categoría debido al mismo efecto, comportándose en iguales proporciones de deposición de tejido adiposo ( 1 y 2 ) en 3/8 CxH y TC de las categorías novillos y novillitos. En esta variable el efecto biotipo demostró mayores diferencias entre novillos $3 / 8 \mathrm{CxH}$ y TC, y 5/8 CxH y TH, que entre cruzas y definidos. En todos los individuos los grados de engrasamiento en las tres categorías ( $\mathrm{T}, \mathrm{NT}$ y NO) resultaron 1 y 2 , niveles adecuados para un buen rendimiento de cortes comerciales sin producir desperdicios en la industria.

Las unidades porcentuales de las categorías están muy influenciadas por el peso vivo de faena, obteniéndose mayor proporción de novillitos cuando éste oscila alrededor de $400 \mathrm{~kg}$ de PV, logrado en los novillos cruzas del ensayo y similar a los informados en novillos Angus ${ }^{6}$.

El efecto biotipo no influyó en la conformación de la res evaluada a través de la tipificación, resultados coincidentes con los de otros autores ${ }^{16}$, no siendo así en todos los casos ${ }^{17}$. Al evaluar diferentes sistemas de alimentación, pasturas y pasturas + concentrados, la conformación fue mejor en el primer caso ${ }^{18}$. Novillos Angus a igual peso de faena pastoreando gramíneas megatérmicas obtuvieron conformaciones inferiores ${ }^{6,7}$. El grado de terminación se encuentra influenciado por el sistema de alimentación, alcanzando en reses de novillos terminados en pasturas grado 1 y $2^{6,18,23}$.

Rendimiento de cortes de diferentes calidades. Los resultados inherentes al peso de los cortes comerciales clasificados de $1^{\circ}, 2^{\circ}$ y $3^{\circ}$ calidad comercial y sus respectivos rendimientos, se informan en Tabla 3.

La suma de los cortes de primera calidad fue $6,2 \mathrm{~kg}$ significativamente superior en $5 / 8 \mathrm{CxH}$ con respecto a TH, mientras que en $3 / 8 \mathrm{CxH}$ y $\mathrm{TC}$ fue de $0,4 \mathrm{~kg}$ sin evidenciarse diferencias significativas, manifestándose el efecto biotipo para esta variable ${ }^{10,21,28}$. Los datos de rendimiento industrial de cortes en la región es otra información útil y escasa para la industria frigorífica, que vende kilogramos de carne sin hueso al mercado interno y externo.
No se encontraron diferencias significativas por efecto del biotipo en los cortes de $2^{\circ}$ y $3^{\circ}$ calidad y tampoco en los rendimientos de las tres categorías de cortes ${ }^{10}$, no coincidiendo con datos obtenidos por otros autores ${ }^{28,29}$. Al comparar tamaños corporales se obtuvieron diferencias significativas en los conjuntos de cortes de diferente destino comercial ${ }^{24}$. Debido a la importancia comercial de esta variable, investigadores han utilizado diferentes parámetros para estudiarla, así el peso del cuarto pistola o rendimiento de los cortes del cuarto trasero son utilizados como estimadores, registrándose diferencias asociadas a la dieta y raza ${ }^{18,22}$.

Se concluye que los cuatro biotipos de novillos de dos años de edad evaluados en este trabajo constituyen opciones viables para obtener buenos rendimientos de res y de cortes comerciales denominados de alta calidad (alto valor comercial), evidenciándose la influencia de la sangre índica sobre la mejoría de estas variables.

\section{REFERENCIAS}

1. Adams J. 2007. La empresa ganadera del NEA: sus debilidades y fortalezas en los últimos 25 años. Rev. Amanecer Rural (Resistencia, Argentina), Junio: 39-43.

2. Anónimo. 2005. Carnes argentinas. Catálogo de cortes. Revista Red Alimentaria (Buenos Aires, Argentina) 9: $17-46$.

3. Arias AA, Slobodzian A. 1994. Evaluación de la condición corporal. Boletín Serie Técnica INTA Corrientes (Argentina) 8: 20.

4. Balbuena O, Gándara FR, Kucseva CD, Stahringer RC, Picallo AB, Carduza J. 2002. Efecto de sangre cebú, edad de faena y maduración sobre terneza en novillos Brangus. Rev Arg Prod Anim 22: 389-399.

5. Balbuena O, Kucseva CD. 2002. Utilización de la semilla de algodón en la alimentación de bovinos para carne. Rev IDIA XXI (Buenos Aires) 2: 69-72.

6. De León M, Bulaschevich MC, Giménez R, Peuser RA, González CL, Cabanillas MA, Boetto GC. 2007. Caracterización de la canal y la carne de bovinos de invernada intensiva. 1. Angus de cría en región templada. Rev Arg Prod Anim 27: 361-362.

7. De León M, Bulaschevich MC, Giménez R, Peuser RA, González CL, Cabanillas MA, Boetto GC. 2007. Caracterización de la canal y la carne de bovinos de invernada 
intensiva. 2. Angus de cría en región subtropical. Rev Arg Prod Anim 27: 363-364.

8. Garciarena D, Teira G, Perlo F, Bonato P, Pasinato A, Monge R, Vittone S, Galli IO. 2004. Encierre terminal y calidad de carnes. 1. Valor de la res. Rev Arg Prod Anim 24: 1.

9. Garcilazo MG, Kugler NM, Barbarossa RA. 2007. Características carniceras y perfil lipídico de novillos en pastoreo de maíz en planta. Rev Arg Prod Anim 27: 379-380.

10. Garriz CA, Mezzadra C, Gallinger MM. 1994. Novillos cruza Limousin y calidad de res. Rev Arg Prod Anim 14: 144.

11. Garriz CA, Gallinger MM, Romero N, Suárez V, Busetti MR, Carduza F, Rivera MI. 1998. Cortes Hilton de novillos puros y cruzas criollo argentino. Rev Arg Prod Anim 18: 262-263.

12. Lowman BG, Scott NA, Somerville SH. 1976. Condition scoring of cattle, East of Scotland College of Agriculture Bulletin, 6 p.

13. Martínez Ferrer J, Teira G, Perlo F, Bonato P, Tisocco O, Ustarroz E. 2007. Fuentes de lípidos en dietas base alfalfa sobre la respuesta animal y la calidad de carne de novillos. Rev Arg Prod Anim 27: 365-366.

14. Oficina Nacional de Control Comercial Agropecuario (Argentina). 1973. Régimen de clasificación y tipificación oficial de las carnes bovinas (tipo y grado de gordura). Res. NJ-378/73. http://www.oncca.gov.ar/principal. php?nvx_ver $=566$.

15. Oficina Nacional de Control Comercial Agropecuario (Argentina). 1973. Escalas de peso limpio para la clasificación y tipificación oficial de carnes bovinas. Res. J-379/73. http://www.oncca.gov.ar/principal.php?nvx_ver=567.

16. Orellana CR, Fumagalli AE, Cornacchione M, Salgado JM, González MA, Oneto C. 2004. Ganancia de peso y características de res de novillos Braford, criollos y cruza índica. Rev Arg Prod Anim 24: 391-392.

17. Orellana C, Peña F, García A, Perea J, Martos J, Domenech V, Acero R. 2009. Carcass characteristics, fatty acid composition, and meat quality of criollo argentino and Braford steers raised on forage in a semi-tropical region of Argentina. Meat Science 81: 57-64.

18. Realini CE, Duckett SK, Brito GW, Dalla Rizza M, De Mattos D. 2004. Effect of pasture vs concentrate feeding with or without antioxidants on carcass characteristics, fatty acid composition, and quality of Uruguayan beef. Meat Science 66: 567-577.
19. Rearte D. 2010. Situación actual y prospectiva de la producción de carne vacuna. http://www.inta.gov.ar/balcarce/ carnes/SituacionActual_Prostpectiva.

20. Rébak GI, Capellari A, Revidatti MA, Zorzoli NM. 2002. Rendimiento de despostada y de cortes comerciales de novillos cruza 2/3 Cebú y 2/3 Hereford, en el norte de la Provincia de Corrientes. Anales XXIII Sesión Comunicaciones Cientificas, Fac Cs Vet UNNE (Corrientes, Argentina) p. 67.

21. Rébak GI. 2007. Atributos de la carne. Rev Amanecer Rural (Resistencia, Argentina), Junio: 53-60.

22. Rochinotti D. 2002. Uso de la suplementación energético-proteica. Rev IDIA XXI (Buenos Aires) 2: 64-68.

23. Rodríguez-Voigt A, Noguera E, Rodríguez HL, Huerta NO, Morón O, Rincón E. 1997. Crossbreeding dual-purpose cattle for beef production in tropical regions. Meat Sci 47: 177-185.

24. Sampedro D, Vogel O, Celser R. 2004. Suplementación de vacunos en pastizales naturales. Boletín Serie Técnica INTA EEA Mercedes (Argentina) 34: 24.

25. Santini FJ, Villarreal EL, Faverin C, Depetris G, Pavan E, Grigera JJ, Grigera JM, Cossú ME, Schor A. 2006. Características productivas, composición de carcasa y calidad de carne de novillos de diferente tamaño estructural alimentados en feed lot con dietas de concentraciones energéticas distintas. Rev Arg Prod Anim 26: 231-244.

26. Schindler V, Pruzzo L, Olivera M, Grigera J, Abbiatti N, De Santa Coloma L. 2004. Predicción de rendimiento de cortes minoristas de reses bovinas en Argentina. Arch Latinoam Prod Anim 12: 105-111.

27. Teira G, Perlo F, Bonato P, Tisocco O. 2006. Calidad de carnes bovinas. Aspectos nutritivos y organolépticos relacionados con sistemas de alimentación y prácticas de elaboración. Rev Ciencia, Docencia y Tecnología (Univ Nac Entre Ríos, Argentina) 33: 173-193.

28. Villarreal EL, Santini F, Faverin C, Depetris G, Paván E, Grigera J, Schor A, Grigera JJ. 2005. Efectos de biotipo y dieta sobre características de carcasa y carne de novillos engordados a corral. Rev Arg Prod Anim 25: 125-126.

29. Villarreal EL, Santini F, Paván E, Faverin C, Depetris G, Grigera J. 2005. Efectos de biotipo y tipo de suplemento sobre características productivas y al sacrificio de novillos en pastoreo. Rev Arg Prod Anim 25: 335-336. 